\title{
X.
}

\section{Ueber die Wirkung der Wasserentziehung auf Thiere.}

Experimentaluntersuchung.

(Aus dem Pathologischen Institut der Königl. Universität zu Palermo.

Dir. Prof. Sirena.)

Von Dr. B. Pernice, und Dr. G. Scagliosi, privatdocenten und Sector ami Path. Inst, $\quad$ Assistenten am Path. Inst.

(Hierzu Taf. V und VI.)

Die gegenwärtig geringen Kenntnisse über die Folgen des Durstes, besonders von anatomisch-histologischer Seite, haben uns zu den folgenden Untersuchungen geführt, welche seit dem Jahre 1891, und zwar im Anschluss an experimentelle Ergebnisse, die der eine von uns mit Dr. G. Alessi in dem Institut des Prof. Sirena erhalten hat, angestellt wurden.

Obgleich wir zuerst beabsichtigten, unsere Aufmerksamkeit direct auf die anatomisch-pathologischen Alterationen zu richten, so haben wir es doch für nützlich gehalten, auch einen Beitrag zu den pathologischen Erscheinungen während des Lebens der Thiere za liefern, speciell in Bezug auf einige Punkte von besonderer Wichtigkeit.

Es steht ausser Zweifel, dass das Wasser das erste, unumgänglich nöthige Nahrungsmittel sowohl der Thiere, wie der Pflanzen darstellt; es spielt eine grosse Rolle bei der Function der thierischen Organismen, was sofort einleuchtet, wenn man bedenkt, dass der Körper gegen 58,5 pCt. (Bischoff, Landois) bis 65,7 pCt. (Volkmann) Wasser in allen seinen Geweben enthält, dass das Blut $\frac{2}{5}(681,63$ pro mille, Landois), dass jeder Absonderungsstoff $\frac{8}{10}-\frac{9}{10}$ Wasser enthält, und die wichtigsten Gewebe, das Gehirn u. s. w., fast $\frac{2}{3}$ Wasser enthalten (Lussana). Wenn man weiter erwägt, dass das Wasser beständig aus dem Organismus durch den Harn, den Stuhl, die Haut und die Lungen ausgeschieden wird, und ebenso, dass für 


die Prozesse der Verdauung und der Resorption eine Auflösung der meisten Substanzen in Wasser nothwendig ist und dass zahlreiche Auswurfsstoffe als wässerige Lösungen den Körper wieder verlassen, so tritt die grosse Bedeutung des Wassers und seines steten Wechsels für den Organismus sofort hervor.

Hoppe-Seyler sagt: alle Organismen leben im Wasser und zwar im fliessenden Wasser, ein Ausspruch, welcher gewissermaassen dem alten: Corpora non agunt nisi fluida entspricht. H. Nothnagel sagt: „Die zu den Gemeingefühlen gerechneten Empfindungen sind jedoch nicht, wie augenblicklich ïblich, sämmtlich zusammenzufassen, sondern meines Erachtens vorläufig in mindestens 2 Gruppen zu zerlegen. Für die eine derselben wäre der bisherige Name beizubehalten, für die andere erlaube ich mir die Bezeichnung Ernährungsgefühle vorzuschlagen. $\mathrm{Zu}$ diesen letzteren wären zu rechnen: Hunger, Durst, Lufthunger".

Die Entbehrung des Wassers muss nothwendig eine Störung im Ablauf der Lebenserscheinungen setzen, indem kein Organismus ohne den Ersatz seines verbrauchten Wassers sich erhalten kann; sein Körpergewicht muss wenigstens zur Hälfte aus Wasser zusammengesetzt sein, dessen Gegenwart unentbehrliche Voranssetzung für den ungestörten Ablauf der Lebensprozesse ist.

Es ist klar, dass die pathologischen Erscheinungen des Durstes intensiver, als diejenigen des Hungers, sein müssen, weil der Hungerzustand bei fortdauernder Wasserzufuhr weit länger ertragen wird. Ein Mensch kann bei vollständiger Aufhebung der Zufuhr von Flüssigkeiten das Leben nur 3-4 (Longet), allenfalls 6-12 Tage (Birch-Hirschfeld), bei fortdauernder Wasserzufuhr dagegen, ohne Genuss fester Substanzen, bis über 40 Tage und noch länger fortsetzen. In der That theilt Lussana einen Fall mit, wo eine Frau nur mit Wassergenuss 72 Tage lebte, und einen anderen Fall, der den Hungerkünstler Succi betrifft, der mit Wassergenuss 40 Tage ohne sensible Störungen leben konnte, was auch von F. Müller, I. Munk, H. Senator, N. Zuntz in ihrem Bericht über die Hungerkünstler Cetti und Breithaupt bewiesen worden ist.

Weitere Beiträge dazu geben die Thierversuche: Die von Redi und Casagrande der Inanition unterstellten geflügelten 
Thiere, die von Chossat ihrer Nahrung beraubten Hunde konnten am Leben bleiben, wenn sie Wasser tranken. Die von Luciani und Bufalini dem Hunger unterworfene Hündin konnte das Leben bei einer Wasserzufuhr von $150 \mathrm{ccm}$ pro Tag 43 Tage erhalten. Laborde stellte Versuche an Hunden von demselben Gewicht und Alter an, an denen er die Bedeutung des Wassers für den Organismus bei der Inanition feststellen wollte. Er entzog dem einen Hunde das Wasser und die feste Nahrung, liess dagegen dem anderen bei gleichen Verhältnissen durchschnittlich $100 \mathrm{~g}$ Wasser täglich darreichen. Der des Wassers beraubte Hund starb am 20. Tage, nachdem or von dem ursprünglichen Gewicht $(15,500 \mathrm{~kg})$ fast die Hälfte, nehmlich $7,500 \mathrm{~kg}$, verloren hatte; der andere Hund, der Wasser trank, lebte bis zum 39. Tage: er hatte zu Beginn des ersten Hungertages ein Gewicht von gerade $15,500 \mathrm{~kg}$, und wog am Ende des Hungers nur $7,600 \mathrm{~kg}$; or erholte sich bald wieder bei fester Nahrungszufuhr.

Man kann also nicht an der hohen Bedeutung des Wassers für den normalen Ablauf der Lebensvorgänge zweifeln. $W_{0}$ belebte Wesen vorhanden sind, muss sich Wasser finden, dessen Mangel früher, als derjenige an festen Nahrungsmitteln, zum Tode in kurzer Zeit führt; es ist deshalb vorauszusetzen, dass der Durst ernste Störungen in den Organen und Geweben setzen muss, was bis jetzt noch nicht bekannt und Hauptgegenstand dieser Arbeit ist.

Nach Tiedemann wird während des heftigen Durstes, wie ihn Reisende in der Wüste empfinden, das Gefühl der Trockenheit im Munde und in der Kehle brennend und schmerzhaft, die Athembewegungen vermehren sich und sind durch Schluchzen und tiefe Athemzüge unterbrochen, die Stimme wird nach und nach belegt, das Sprechen erschwert, der Puls beschleunigt, die Haut trocken, spröde und brennend heiss, die weisse Augenhaut zeigt sich geröthet, die Muskeln werden matt, jede Bewegung ist kraftlos und träge; sodann wird der Verdurstete wahnsinnig, manchmal wüthend, worauf ein höchster Grad von Entkräftung, ein höchst brennendes Gefühl in der Kehle, Ohnmacht und Verlust des Bewusstseins folgen.

Der Leichenbefund Verdursteter zeigt, neben Schwund des 
Fettgewebes und hochgradiger Abmagerung der Musculatur, auffällige Atrophie und Trockenheit der Unterleibsorgane, alle Seund Excrete und das Blut sind eingedickt, endlich constatirt man zahlreiche Entzündungen und manchmal partiellen Brand einiger Unterleibsorgane, so dass der Durst wie eine entzündliche Krankheit tödtet (Longet).

Um die Folgen des Durstes von denjenigen des Hungers vollständig zu trennen, haben wir Versuche an Vögeln angestellt, welche weniger, als Säugethiere, das Eruährungsgefühl des Durstes empfinden, indem ihr Organismus weniger Wasser bedarf. Pernice und Alessi haben bewiesen, dass die verlängerte Wasserentziehung bei Hunden eine unzureichende Ernährung derselben nach sich zieht, indem diese Thiere bei ungelöschtem Durste keine Nahrung mehr zu sich nehmen; in der That fressen diese verdursteten Thiere weniger, nehmen nur Brodkrume und weisen selbst diese zurück, wenn sie trocken ist, so dass sie endlich in vollständiger Inanition zu Grunde gehen. Die Sache verhält sich ganz anders bei Tauben und Hühnern, welche, des Wassers beraubt, bis an's Lebensende fressen, obgleich nicht so reichlich, wie gewöhnlich und früher. Th. Chossat bemerkte bei den des Wassers beraubten Fröschen ausser Störungen der Circulation und der Athembewegungen eine Herabsetzung des Empfindungsvermögens und tetanische Contractionen der Muskeln; die Frösche starben, nachdem sie 35 pCt. des Körpergewichts eingebüsst hatten. Falk und Scheffer, dann Scheffer allein, stellten an Tauben Versuche über den Tod durch Durst an: von diesen Thieren starb das eine am 13. bis 14. Tage und verlor $127,4 \mathrm{~g}$ von seinem Anfangsgewicht (286,4); das zweite starb am 13. Tage, nachdem das Anfangs. gewicht $(320.4 \mathrm{~g})$ eine Abnahme von $151,4 \mathrm{~g}$ erfahren hatte.

Schuchardt wählte auch Tauben, welche mit an der Luf getrockneter Gerste ernährt wurden, wobei zu merken ist, dass di Luft zur Zeit seiner Versuche feucht war. Die 6 von ihm benutzte Thiere starben durchschnittlich am 10. Tage; alle Tauben verlores beträchtlich am Gewichte (die Gewichtsabnahme war auffallend)

v. Voit stellte Versuche über den Wassergehalt der Muskel und Nerven der an Cholera gestorbenen Menschen an, und fanc dass er um 5-6 pCt. abgenommen hatte. 
Gürbe studirte den Einfluss der Wasserentziehung auf die quantitativen Störungen der rothen Blutkörperchen bei Fröschen; neuerdings, als wir gerade mit unseren Versuchen beschäftigt waren, theilte F. Nothwang seine Beobachtungen über die Folgen des Durstes mit. $\mathrm{Er}$ experimentirte, wie Falk und Scheffer, an Tauben, welche schon nach dem 2. Tage Störungen ihres Gesundheitszustandes zeigten; die Thiere starben durchschnittlich nach $4 \frac{1}{2}$ Tagen. Der Leichenbefund war bei allen Thieren gleich, was eben von Falk und Scheffer constatirt worden war: die Muskeln noch wohl erhalten, von dunkelrother Farbe (Spickgansfarbe), ihre Schnittfläche hatte den feuchten Glanz eingebüsst, die Sehnenhäute waren schwer abzulösen und von grauer Farbe, das subcutane Fettgewebe hatte wenig abgenommen und war trocken, auch die Speiseröhre und der innere Theil des Kropfes waren trocken.

Die von Nothwang gemachten Analysen ergaben eine Concentration der Gewebe; die Thiere starben, als die Trockenheit der Organe $33,47 \mathrm{pCt}$. betrug und der Wassergehalt bis auf 66,53 pCt. gesunken war. Der Tod durch Durst hängt, dem Verfasser nach, nicht nur von der Wasserberaubung, sondern auch von der Zurückhaltung der Zersetzungsprodukte ab, indem der Organismus normal durch verschiedene Se- und Excrete sich von diesen letzteren befreit, wozu er jetzt nicht mehr befähigt ist. Die chemische Analyse der an Durst zu Grunde gegangenen Tauben bewies in der That, dass die Absonderungsprodukte mehr Bestandtheile, als im normalen Zustande, enthielten.

Wir hatten den Plan gefasst, die functionellen Störungen und insbesondere die anatomisch-pathologischen Veränderungen der verschiedenen Gewebe zu studiren. Zu diesem Zwecke haben wir junge Hühner gewählt, welchen wir das Trinkwasser ganz entzogen, während sie mit trockenem Mais gefüttert wurden. Um aber einen Vergleich anstellen zu könuen, haben wir es für zweckmässig gehalten, auch einen Hund in Beobachtung zu nehmen, bei welchem, obgleich der Tod bei vollständiger Inanition eintrat, die erhaltenen Ergebnisse bei gleichzeitiger Wasserberaubung doch Unterschiede zeigten, im Vergleich zu denjenigen, welche in der Inanition bekannt sind.

Die Hauptergebnisse unserer Versuche haben wir in einer vorläufigen Mittheilung zusammengefasst (Riforma medica. 1893). 
Jedes Versuchsthier wurde, nach mehreren Tagen einer vorhergegangenen Beobachtung, wobei es reichliche Fütterung und Wasser nach Belieben bekam, allein in einem Hühnerkorbe gehalten, und vollständig des Wassers beraubt. Der Hund bekam nur trockenes Brod, die Fütterung der Tauben bestand aus trockenem Mais.

Zuerst wurden die Thiere im normalen Zustande untersucht, dann in dem der Wasserinanition bis zum Tode. Unsere Untersuchungen während des Lebens betrafen das Körpergewicht, die Temperatur und das Blut, den Harn nur bei dem Hunde. Wir öffneten die Leichen immer bald nach stattgehabtem Tode. Nach vorgängiger makro- und mikroskopischer Untersuchung der frischen Organe wurden kleine handliche Gewebsstückchen in eine recht grosse Quantität der Müller'schen Augenflüssigkeit oder in Flemming'sche Lösung (in diesem Falle dünnere Stückchen, 2-4 mm dick), je nach dem $Z$ wecke der mikroskopischen Untersuchung und je nach dem Gewebe, eingelegt. Nach vollendeter Härtung wurden die Stücke, wie üblich, in Paraffin oder in Celloidin eingebettet und dann geschnitten. Als Färbungsmittel benutzten wir Alaun-, Borax-, Pikrolithioncarmin, Böhmer'sche Hämatoxylin-, Eosin-Hämatoxylinlösung; wir wandten auch, je nach dem $Z_{\text {wecke der Untersuchung, specielle Färbmethoden }}$ (Golgi, Weigert-Pall, Ranvier-Schultze, A. Fraenkel, Stroebe, Rosin) an.

Um vorsichtig zu Werke zu gehen, haben wir einen normalen Hund und eine gesunde Taube, beide beinahe von demselben Gewicht und Alter, getödtet und aus deren Organen mikroskopische Präparate unter Anwendung derselben Härtungs- und Färbungsmethoden hergestellt, damit wir, falls wir über etwas im Zweifel wären, die Ergebnisse der mikroskopischen Untersuchung der Organe der verdursteten Thiere controliren konnten.

\section{Experiment am Hunde.}

Wir haben einen Hund von $6700 \mathrm{~g}$ Körpergewicht, dessen Temperatur 2.wischen $38,9^{\circ}-39,1^{\circ}$ C. schwankte, am 15. September 1891 um 9 Ubr des Wassers beraubt, und beauftragten den Laboratoriumsdiener, dem Versuchsthiere nur seit 24 Stunden gebackenes Brot darzubieten. In den folgenden Tagen frass das Thier wenig und von dem 9. Tage an rührte es die dargebotene Nabrung nicht mehr an. Es starb am 27. desselben Monats, also nach ungefähr 
11 Tagen. Das Gewicht fiel von Tag zu Tag, wie selbstverständlich, und betrug am Ende des 11. Tages nur $5075 \mathrm{~g}$, so dass es in 11 Tagen im Ganzen $1625 \mathrm{~g}$, d. h. 24,25 pCt. rom Anfangsgewicht eingebüsst batte. Die Körpertemperatur im Rectum fiel nach und nach bis $37^{\circ} \mathrm{O}$.

Während der Lebenstage bemerkten wir gradweisen Verlust der Kräfte im Allgemeinen, bis einige Tage vor dem Tode die Schwäche so bedeutend wurde, dass das Thier nicht mehr steben konnte. Bei Betrachtung der Augen fiel eine Spur von Gelbfärbung des Weissen vom Auge auf. In den letzten Tagen litt er an einer eiterigen Bindehautentzündung zuerst des rechten Auges, später beider Augen.

Die Respiration zeigte Veränderungen im Rbythmus der Bewegungen. In den ersten Versuchstagen war die Dauer der Inspiration kürzer, als die der Exspiration, deren Zeit sich aber mehr als normal verlängert, zeigte; weiter wurde eine Beschleunigung und Vertiefung der Athemzüge wahrgenommen. An diese schloss sich endlich vor dem Tode eine angestrengte und mühsame Thätigkeit aller Respirationsmuskeln, nacbher wurde die Athmung nach Zahl und Tiefe der Bewegung beschränkt, es erfolgten weiterhin nur noch wenige schnappende Züge, dann ruhten die Muskeln völlig und das Thier starb. Der Puls unterschied sich vom 6. Tage an von dem während der Nahrungsperiode dadurch, dass er schnell und klein wurde.

Während das Thier am letzten Trinktage $265 \mathrm{ccm}$ Harn ausgeschieden hatte, sank die Harnmenge am ersten Dursttage sofort herab, um unter allmählichen Schwankungen am 11. Tage bis auf $25 \mathrm{ccm}$ zu sinken. Im Ganzen wurden $419 \mathrm{ccm}$, also irm Mittel $38 \mathrm{ccm}$, für jeden Dursttag ausgeschieden. Der Harn enthielt einen leichten Ueberschuss an Phosphaten und in den letzten Tagen Eiweiss, allerdings nur in sehr kleiner Menge, nehm$\frac{1}{2} \mathrm{~g}$ pro mille, hyaline und Epithelcylinder und in fettiger Degeneration begriffene Epithelien im Sedimente. Die Acidität des Harnes nahm, von Beginn des Durstes an gerechnet, zu. Sie war in Luciani's Versuch geringer, offonbar weil Succi alkalische Brunnenwăsser getrunken batte. Das specifische Gewicht des Harns stand in directer Beziehung zur Harnmenge, caeteris paribus stieg es mit sinkender Harnmenge. Es schwankte bei unserem Hunde an den ersten 6 Dursttagen zwischen 1038-1041; die grösseren Werthe fanden sich bei kleinerer Harnmenge. In den letzten Tagen waren wir, wegen der geringeren Harnmenge, nicht mebr im Stande, das specifische Gewicht zu constatiren. Der Harn war frei von Gallenfarbstoffen, dagegen enthielt or bei sorgsamster Untersuchung Traubenzucker und in den letzten Tagen Schleimeiter. Der Harnstoff, dessen Bestimmung nach dem Esbach'schen Verfahren geschab, nabm immer mehr ab, indem er der quantitativen Abnahme des Harns folgte: von $2,50 \mathrm{~g}$ pro Tag, $=59,65$ pro mille, sank er bis zum Tage vor dem Tode auf 0,40 in 24 Stunden, also 16,5 pro mille, herab.

Die Untersuchung des Blutes wurde mit dem Bizzozero'schen Cromocitometro unternommen. Behufs der Zählung wurde der Zählapparat von Thoma-Zeiss angewandt. Die rothen Blutkörperchen und der Hämoglobin- 
gehalt zeigten eine Zunahme. Die Zahl der rothen Körperchen stieg von 5792000 am 1. Tage bis auf 7378000 am 6. Dursttage. Die Zahl der Leukocyten dagegen war während der ersten Dursttage geringer, als in der Norm; dann folgte eine Abnahme der rothen Blutscheiben und der Menge des Hämoglobins, und in den letzten 2 Tagen vor dem Tode trat eine deutliche Leukocytose hinzu.

Die Resultate dieser Analysen sind in folgender Tabelle zusammengestellt, um so dem Leser die Möglichkeit zu geben, ein Urtheil über die Schwankangen der Harnmenge u. s. w. zu gewinnen:

T a b e 11 e I. (Hündin.)

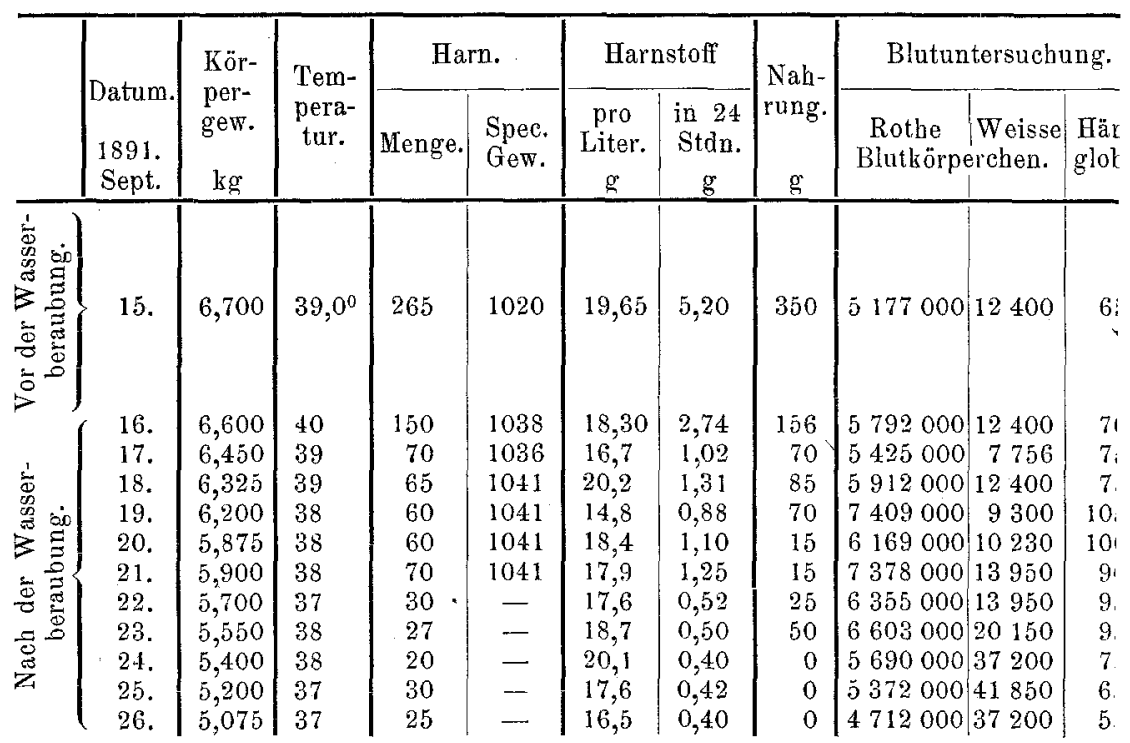

Am 27. Sept. früh Morgens stirbt das Thier.

Bald nach dem erfolgten Tode wurden die Thierleichen obdicirt. Der Befund der Obductionen, um es kurz zu fassen, ist folgender: allgemeine Abmagerung, Muskeln trocken, blass; Fettgewebe trocken, theilweise verschwunden, besonders im Netze. Im Gehirn, Rückenmarke und in den Nerven bemerkt man schon mit blossem Auge starke Congestion. Die Herzbeutelblätter zeigen sich trocken, keine Spur von Flüssigkeit ist im Pericardialsacke vorhanden. Das Brustfell ist scheinbar normal. Die Lungen sind blass, anämisch; dies tritt besser in den Alveolen des vorderen Theiles des Oberlappens hervor, wo 
die Lungen gross, die Ränder gedunsen, abgerundet erscheinen. Sie lassen im unteren Lappen auf der Schnittfläche, besonders auf Druck, eine kleine Menge feinschaumiger Flüssigkeit austreten. Die Bronchien zeigen eine mit einer etwas vermehrten Schleimsecretion einhergehende Schwellung und Röthung ihrer Schleimhaut. Die Milz trocken, klein, ihre Kapsel runzelig, etwas verdickt; auf der Schnittfläche erscheint die Pulpa schlaff, von braunrother Farbe, es treten die Trabekeln relativ stärker hervor, als dies bei normaler Milz der Fall ist. Die Nieren sind von normaler Grösse, hyperämisch, und bieten eine leicht höckerige Oberfläche. Nach Ausführung des grossen Längsschnittes lassen die beiden Nierensubstanzen keine deutliche Aenderung in ihren Grössenverhältnissen erkennen. An der Nierenrinde unterscheidet man rothe Streifchen und Pünktchen; welche mit Streifchen von gelbweisslichgrauer Farbe abwechseln. Der Magen enthält kleinste Mengen von Galle, er ist zusammengezogen und durch Faltenbildung stark verdickt. Durch einen in der Querrichtung der Falten ausgeübten Zug werden diese letzten ausgeglichen. Die Magenschleimhaut in der Nähe der Cardia ist blass; in der Nähe des Magenpförtners (Pylorus) besitzt sie eine rothe Färbung und ist mit einer dünnen Lage von Schleim bedeckt. Die Schleimhaut zeigt überdies viele ganz flache oberflächliche Substanzverluste, meistens auf den Faltenhöhen gelegen, welche eine rundliche oder auch längliche $\mathrm{Ge}$ stalt haben; in diesem letzten Falle stimmt ihre Längsrichtung mit derjenigen der Falten überein. Im Grunde dieser Defecte findet man kleine bräunliche Massen, welche von Blut herrühren und sich leicht abkratzen lassen. Diese Schleimhantgeschwüre, besser hämorrhagischen Erosionen, finden sich sparsam in der Cardiagegend. Die Gedärme sind ganz leer, die Schleimhaut des Duodenums erscheint gallertig. Um die Peyer'schen Haufen findet man congestive entzündliche Hyperämie. Die Leber ist hyperämisch und zeigt eine leichte Verdickung und weissliche Trübung ihrer Kapsel. Auf dem Durchschnitte erscheinen die Acini durch eine graue Zone getrennt. Die Gallenblase enthält wenig dicke Flüssigkeit. Die Harnblase ist vollständig leer, ihre Schleimhaut hyperämisch. Die Bauchspeicheldrüse atrophisch. Die Hoden, die Nebenhoden und die Prostata erscheinen trocken, 
atrophisch. Die Samenleiter und Samenbläschen sind anämisch und fast vollständig leer von Samen.

Wir lassen die Beschreibung der mikroskopischen Befunde folgen.

Im Gehirn und in seinen Häuten bemerkt man vor Allem eine starke Congestion der Blutgefässe, so dass nicht nur die grossen Gefässe erweitert und mit Blut gefüllt erscheinen, sondern auch das reiche Capillarnetz durch eine Art natürlicher Injection sehr deutlich wird. Die Nervenzellen der 4 Schichten and die markhaltigen Nervenfasern des Gehirns sind im Allgemeinen trübe, nehmen die Carminfarbe nicht mehr gut an, manche sind deutlich atrophisch. Die Nervenfasern sind nach der Weigert-Pall'schen Methode nicht mehr so gat zu färben, als die des gesunden Hundes. Im kleinen Gehirn findet man starke Congestion and secundäre Veränderung der Nervensubstanz. Die Congestion der Blutgefässe betrifft mit Vorliebe den oberflächlichsten Theil der Molekularschicht und die Schicht der Purkinje'schen Zellen.

Die starke Congestion der Blutgefässe im Rückenmark lässt die directe Beziehung der Capillaren za den Ganglienzellen erkennen. Diese sind von einem mehr oder weniger vollständigen Gefässringe eingeschlossen; durch die Erweiterung der Gefässe konnte ein gewisser Druck auf die Ganglienzellen ausgeübt werden. Mit dieser Congestion bemerkt man eine Verdickung des Nervenkittes. Die Nervenfasern sind in verschiedenem Grade atrophisch; dies fällt besonders in der Nähe der grauen Substanz auf, welche eine dentliche Abnahme der Zahl und Grösse der noch vorhandenen Ganglienzellen wahrnehmen lässt. Diese Atrophie tritt mehr in den vorderen Hörnern hervor, wo sie so weit fortgeschritten ist, dass manche Zellen zum Schwunde gebracht worden sind; an ihrem Platze nimmt man Höhlen wahr, welche fettige Granula enthalten. Der Nervenkitt dieser Gegend zeigt sich auch verdickt. Der Centralkanal ist erweitert.

Die Fasern des N. ischiadicus (Färbung mit Weigert'scher Methode und Alauncarmin) zeigen wenige Veränderungen; diese bestehen in einer Verdickung des Perineurium und partiell des Eudoneurium. Die von diesem letzten sich abzweigenden Blätter, die Fibrillenscheiden, sind auch etwas verdickt; dies 
tritt besser in die Erscheinung, wo die Blatgefässe erweitert und mit Blut gefüllt sind. In dem hyperplastischen Bindegewebe bemerkt man eine geringe Rundzelleninfiltration. Die kleinen Arterien haben eine Verdickung ihrer Wände erfahren, die Intima zeigt Reichthum an Kernen. Die Nervenfasern zeigen im Vergleich mit den normalen eine Verminderung ihrer Grösse; einige haben eine weniger intensive Färbung, als die gesunden.

Herz und Muskeln. Im Herzen bemerkt man eine Entzändung der Venen, welche in der Intima mehr ausgeprägt ist. Das die Gefässe umgebende Bindegewebe ist sehr verdickt und an Kernen und kleinen spindelförmigen Zellen reich. Die Muskelfasern sind blass, weniger intensiv gefärbt, ein wenig homogen aussehend, und $z$ war in dem Sinne, dass ihre Streifung weniger angedeutet ist, als normal. Die Fibrillen sind dünner, deshalb erscheinen sie weiter von einander gelagert; ihre Kerne sind zahlreich und einige zeigen Mitosen.

In den Muskeln des Relationslebens bemerkt man die nämlichen, so eben geschilderten Veränderungen, hier aber erscheinen die Alterationen der Gefässhäute noch deutlicher.

Lungen. Die Gefässe der Alveolenwände und der Bronchien sind stark mit Blutkörperchen gefüllt; um sie herum bemerkt man eine mehr oder weniger breite Zone von Bindegewebssklerose und an manchen Stellen ist noch eine kleine rundzellige Infiltration vorhanden. Die Congestion der Gefässe ist so bedeutend und so diffus verbreitet, dass das Capillarnetz deutlich erscheint. Die Alveolen enthalten rothe Blutkörperchen, von denen einige frei im Lumen, andere dagegen zwischen sehr dünnen Fibrinfäden, welche meistens einen Theil des Alveolus einnehmen, liegen. Manche Alveolen sind über die Norm ausgedehnt und es haben sich in ihren Wänden atrophische Zustände entwickelt, d. h. eine Vereinfachung des Lungengewebes. Die Scheidewände sind atrophisch.

Die Bronchien, sowohl die kleinen, wie die mittleren, zeigen sich verändert. Die Bronchialmucosa ist von einem kleinzelligen Infiltrat durchsetzt, ihre Epithelzellen sind stellenweise abgehoben und nehmen nicht mehr gut die Farbe an. Die auf diese Weise desquamirten Epithelzellen finden sich mit Exsudat 
gemischt, welches aus durch Verschleimung zu Grunde gegangenen Zellen besteht. Das Exsudat enthält noch Leukocyten und einige rothe Blutkörperchen. Die weissen Zellen zeigen in ihrem Innern schwarze Pigmentkörnchen.

Milz. Die Kapsel ist verdickt und von grauer Farbe. Die strangförmigen Eortsetzungen treten da, wo sie von der Kapsel ausgehen, in Folge der Pulpaatrophie deutlich hervor und sind hyperplastisch. An den Stellen, wo die hyperplastischen Trabekeln von der Kapsel abgehen, sind kleinste Vertiefungen vorhanden, welche durch stärkere Atrophie der umgebenden Pulpa ihre Entstehung erkennen lassen. Die Pulpa ist übrigens bedeutend atrophisch, die Follikel erscheinen daher scharf abgegrenzt. Einen weiteren Befund bilden partielle, kleine, subcapsulare Hämorrhagien. Viele Zellen befinden sich in mitotischer Theilung.

Niere. Im frischen Zustande, vor der Härtung, haben wir Blut sowohl in den Kapseln, wie in den gewundenen und geraden Kanälchen gesehen. Die Epithelien zeigen sich trübe, körniger, geschwollen und mit undeutlichen Kernen versehen, einige enthalten kleinste Fettkörnchen. Die so eben erwähnten Circulationsstörungen sind besser in Schnitten aus gehärteten Stücken wahrzunehmen. An Stellen, wo der Kapselraum eine relativ grössere Menge von Blut enthält, sieht man das Malpighi'sche Körperchen zur Seite geschoben und unterdrückt, manchmal sogar ganz mit Blut bedeckt. Manche Harnkanälchen sind so stark mit Blut gefüllt, dass die Epithelzellen nicht mehr gut zu sehen sind. An manchen Glomeruli kann man eine mächtig entwickelte Epithelbekleidung auf den Gefässschlingen sehen, welche auf eine Wucherung des Epithels zurückzuführen ist; in anderen bemerkt man, dass die peripherisch gelegenen Glomerulusschlingen von ihrem Epithel entblösst sind oder dass einzelne Zellen sich kolbenförmig abheben; auf diese Weise entstehen die sichtbaren kernlosen Glomeruluscapillaren. Manche Glomerulusepithelien zeigen sich hochgradig verfettet (Osmiumsäure). Im Anschluss hieran hat sich in der Umgebung einzelner Glomeruli und Harnkanälchen eine kleinrundzellige Infiltration des nächst gelegenen Bindegewebes eingestellt. Manche Harnkanälchen sind hie and da mit blutigen oder hyalinen Cylindern ausgefüllt (Schnitte aus gekochten Stücken). Die Epithelzellen 
zeigen Wucherungen, welche wohl die Regeneration des gesetzten Verlustes bezwecken.

In der Leber ist das Bindegewebe zwischen den Läppchen vermehrt. Die Verzweigungen der Pfortader, d. h. die Venen, welche im interlobularen Gewebe verlaufen, zeigen Erweiterung ihres Lumens und Veränderungen der Intima, welche aus einer stark gefärbten kernreichen Schicht besteht. Die Gallengänge sind auch erweitert, einige lassen eine grössere und höhere Zellenbekleidung wahrnehmen. Das Cylinderepithel der Gallencapillaren ist an einigen Stellen abgestossen; die daraus sich ergebenden Zellenlamellen bilden allerlei gekrümmte Falten und erfüllen das Lumen des Gallenganges. Die Zellen dieser Falten zeigen eine schwache Carminfärbung, wenn man sie mit dem Färbungsvermögen jener Zellen vergleicht, welche sich an ihrem rechten Platze befinden und sich noch intact zeigen. Das interlobulare Bindegewebe ist hyperplastisch und Sitz einer sehr geringen Durchsetzung mit Rundzellen. Stauungshyperämie ist in allen venösen Gefässen wahrnehmbar. Die Lebervenen bieten eine Verdickung ihrer Wand. Die Leberzellenbalken sind mehr oder weniger verschmälert, so dass der Raum zwischen den Capillaren weniger von Leberzellen eingenommen wird. Die Leberzellen bieten im Allgemeinen eine normale Anordnung, sie haben aber im Umfange so abgenommen, dass die Maschen des Capillarnetzes besser hervortreten. Die Zellen dieser Balken zeigen sich, besonders um die Vena centralis herum, am meisten verändert, sie sind kleiner, körniger, fettig degenerirt und nehmen nicht mehr gut die Carminfarbe (Alaunoarmin) an. Man findet wenige Mitosen.

Magen und Darm. Man bemerkt im Magen eine starke congestive Hyperämie, welche die oberflächlichsten Gefässe erkennen lässt. An manchen Stellen ist das Schleimhautgewebe hämorrhagisch infiltrirt und braunroth gefärbt. Diese Blutungen entsprechen meistens den Stellen, wo man einen Defect in der Schleimhaut des Magens beobachtet Diese ganz flachen, oberflächlichen Substanzverluste, meistens auf den Faltenhöhen gelegen, haben eine rundliche oder häufig auch längliche Gestalt; sie betreffen nur die Epithelzellen und manchmal die Drüsen. Im Grunde dieser Defecte, wie auf der Mucosa, findet 
man ein durchsichtiges, fettige Körner und eine reichliche Menge von Rundzellen enthaltendes Exsudat. Das Gewebe zwischen den so zahlreichen Drüsen der Tunica propria erscheint vermehrt und von jungen Zellen, sog. Rundzellen, durchsetzt. Diese kleinzellige Infiltration tritt auch im Gewebe unter den Drüsen, in der Submucosa, um die Gefässe herum und zwischen den glatten Fasern der Muskelhaut hervor. Die Mucosa zeigt hie und da eine Atrophie der Drüsen, welche weiter aus einander stehen, und der so entstandene Raum wird von hyperplastischem Gewebe eingenommen. In der Regio pylorica sind manche Drüsen erweitert, ihre Auskleidung von Cylinderzellen ist höher als normal; andere zeigen in ihrem Innern Lymphzellen, so dass sie das Aussehen kleiner beginnender Lymphknötchen gewinnen; andere endlich erscheinen auf Querschnitten von einer kleinrundzelligen Infiltration umgeben. Im Allgemeinen ist das Drüsenepithel, sowohl das der Regio cardiaca, als das der Regio pylorica, mehr homogen als normal, kleiner, vón Vacuolen durchsetzt; es nimmt nicht mehr gut die Farbe an. Man bemerkt noch weiter symmetrische und asymmetrische Karyokinesen (Behandlung nach Elemming), and zwar stammen sie besonders aus dem Epithel der Fundusdrüsen.

Die entzündlichen Veränderungen im Duodenum sind von derselben Art und Intensität, wie die für den Magen beschriebenen, aber hier herrschen die Hämorrhagien vor, welche mit Vorliebe die Submucosa und die Muskelhaut betreffen. Diese Hämorrhagien werden durch Heerde von verschiedenem Durchmesser gebildet. Neben diesen Alterationen bemerkt man in den Drüsen Cysten von verschiedener Gestalt und Grösse, deren Inhalt aus lymphoiden Zellen, länglichen hydropischen Zellen und einer in's Gelbliche fallenden Masse besteht. Die Wand dieser cystischen Erweiterungen ist mit glattem Epithel versehen, dessen Zellen ein glasiges Protoplasma und einen schlecht gefärbten Kern darbieten. In manchen Lymphknötchen findet man kleine umschriebene Hämorrhagien, in anderen dagegen beobachtet man kleine unregelmässige Aushöhlungen, mit geschwollenen und durchsichtigen kleinen Zellen gefüllt.

Die Mitosen, besonders in den Lieberkühn'schen Drüsen, sind zahlreich. 
Alle bis jetzt beschriebenen Veränderungen im Duodenum nehmen in demselben Maasse ab, als man im Darme selbst von dem Duodenum zum Dickdarm herabsteigt. Dabei findet man congestive Hyperämie und Atrophie der Drüsen, von denen einige ein netzförmiges Aussehen darbieten, nachdem ihre Zellen theilweise verloren gegangen sind und so ihr Gerüst deutlicher erscheint. Man sieht zahlreiche Mitosen.

Im Pankreas wiederholen sich die Circulationsstörungen. Neben Hyperämie beobachtet man eine Atrophie der. cylindrischen Zellen und eine leichte Vermehrung des Zwischenbindegewebes. Der hellere peripherische Abschnitt der Zellen zeigt sich grösser, als der körnige, welcher stellenweise kaum angedeutet ist.

In Uterus und Eierstöcken bemerkt man ausser starker Congestion Atrophie der Zellen, welche kleiner, blass und weniger gefärbt erscheinen.

Lymphknoten. Die Schnitte zeigen eine Füllung zahlreicher Gefässe. Diese Hyperämie erstreckt sich auf alle Theile der Drüsen. Die Lymphkörperchen sind weniger zahlreich, und zwar tritt dies besonders in der Marksubstanz hervor, so dass die Septa dicker erscheinen und das Reticulum zum Vorschein kommt.

Die Knochen zeigen nur eine mässige Stauungshyperämie.

\section{Versuche an Hühnern.}

Drei junge Hühner, die während einiger Tage im Laboratorium reichliche Fütterung nach Belieben bekommen hatten, wurden nach einander des Wassers beraubt und mit einer grossen Menge von trockenem Mais gefüttert. Die in 24 Stunden verbrauchte Quantität von Mais wurde berechnet, indem man den Rest wog.

An dem 1. Huhn, welches ein Anfangsgewicht von $665 \mathrm{~g}$ hatte, haben wir vom 25. September 1891 an unsere Untersuchungen angestellt. Das Thier starb den 4 . des folgenden Monats, als es $440 \mathrm{~g}$ wog, nachdem es alsó $225 \mathrm{~g},=33,83 \mathrm{pCt}$. vom Anfangsgewicht eingebüsst hatte, und als zugleich die Körpertemperatur bis $40^{\circ} \mathrm{C}$. gesunken war.

Die Untersuchungen am 2. Huhn von $550 \mathrm{~g}$ Körpergewicht wurden am 10. Juni 1892 begonnen; dies Thier starb am 18. desselben Monats, also nach ungefäbr 8 Tagen. Das Gewicht fiel von Tag zu Tag und betrug am Ende des 8. Tages $358 \mathrm{~g}$, so dass es in dieser Zeit im Ganzen $210 \mathrm{~g}$, $=38,18$ pCt. vom Anfangsgewicht, eingebüsst hat.

Das 3. Thier von $633 \mathrm{~g}$ Körpergewicht haben wir am 30. September 
1892 des Wassers beraubt; es starb am 10. October, nachdem es $261 \mathrm{~g}$, also $41,23 \mathrm{pCt}$. von seinem Anfangsgewicht verloren hatte. Die Körpertemperatur stieg bis $40,4^{\circ} \mathrm{C}$.

Alle 3 Thiere frassen während der Beobachtungstage nicht viel weniger, als gewöhnlich, und zwar bis kurz vor dem Tode; ihre Körpertemperatur und ibr Gewicht fiel von Tag zu Tag, sie saben seit den ersten Tagen leidend aus, waren unrubig und suchten die Käfigthür. Am 4.-5. Tage fingen sie an matt und traurig zu werden, ihr Anfangs rother Kamm begann vom 4. Tage an zu erblassen, wurde dann cyanotisch, worauf es schwierig war, aus dem Kamm einen Tropfen Blut zu entziehen, denn dieses floss spät heraus. In den letzten Lebenstagen konnten die Thiere nicht mebr gut stehen, sie kawen nur für einige Minuten auf die Füsse, wenn man sie mit den Händen erbob, endlich kurz vor dem Tode konnten sie auch mit dieser Hülfe nicht mehr stehen.

Nach vollkommener Erschöpfung und bedeutender Abmagerung, bei auffallender Trockenheit der Gewebe, schwerem Athmen und ausgesprochener Cyanose gingen die Thiere zu Grunde: das erste, wie gesagt, am 9., das andere am 8. und das dritte am 10 . Tage nach der Wasserberaubung.

Die Blutuntersuchung, welche mit dem Globulimeter und Fleischl'schen Hämometer ausgeführt wurde, zeigte eine Vermebrung der rothen Blutkörperchen und des Häıoglobins, weiterhin eine leichte, nicht constante Zunahme der weissen Blutzellen.

Wir haben in der nebenstehenden Tabelle die Resultate der täglichen Beobachtungen zur Anschauung gebracht, damit jederman dieselben übersehen kann.

Bei der Obduction aller drei Thiere haben wir Veränderungen in verschiedener Intensität beobachtet. Der Kamm, die Mundschleimhaut, die Haut boten eine dunkelrothe, fast schwarze Farbe dar. Das Fett war beinahe vollständig geschwunden, die Gewebe zeigten sich trocken, das aussebliesslich in den Venen und im rechten Herz enthaltene Blut war schwarz und dick. Es bestand Stauungshyperämie in allen Organen, besonders in der Leber, den Nieren und im ersten Theil des Verdauungstractus, einmal auch im Dickdarm. Der Kropf enthielt beständig, besonders bei dem ersten und dritten Versuchsthiere, Maiskörner; wenig grünliches, mit Steinchen vermischtes Material befand sich im Magen.

\section{Histologische Untersuchung.}

Gehirn (Färbung mit Pikrolithioncarmin, Alauncarmin und Weigert-Pall'scher Methode). Die Pia mater zeigt sich leicht 


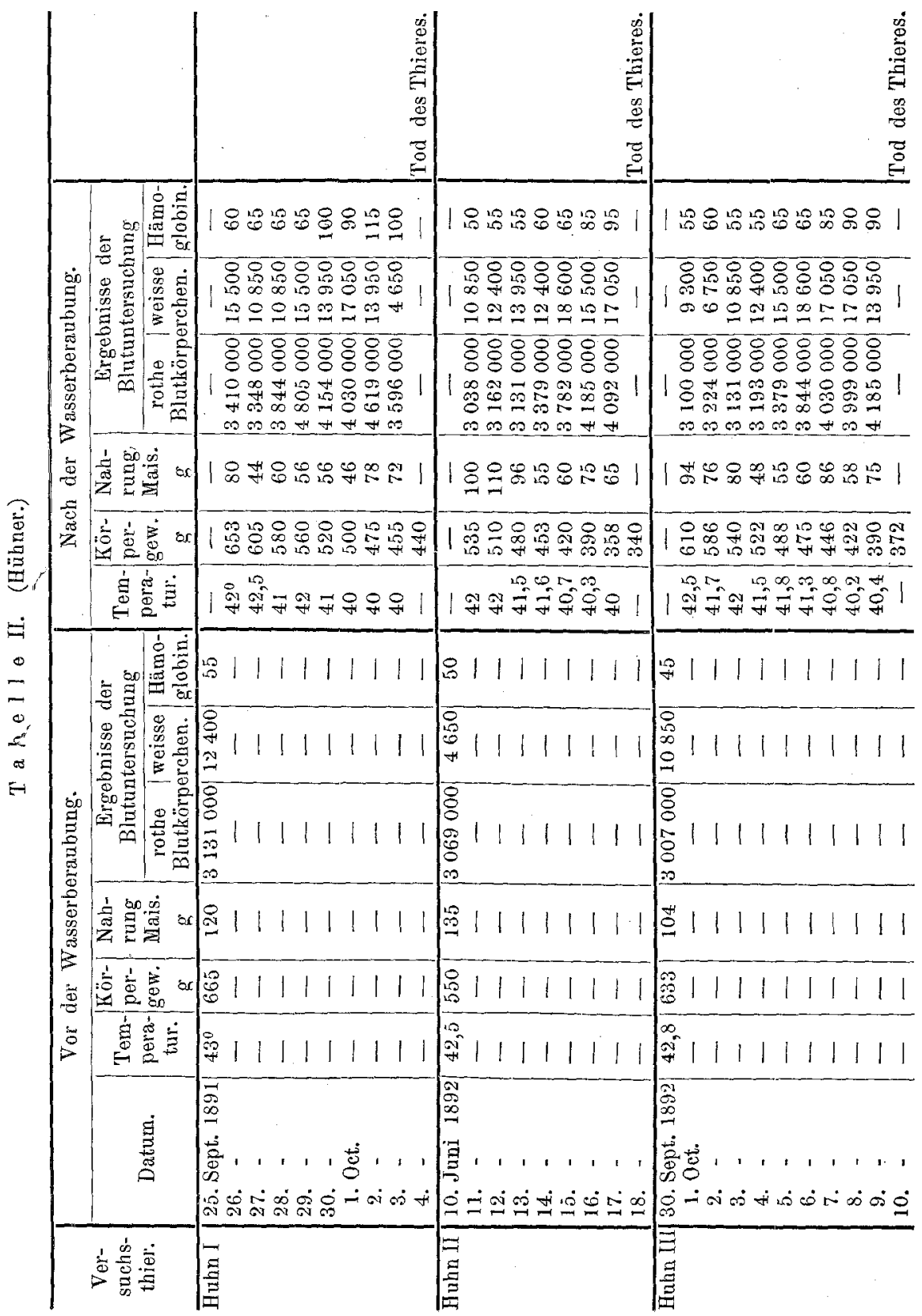


verdickt und mit sog. Rundzellen hie und da infiltrirt. In der Gehirnsubstanz bemerkt man starke Hyperämie, welche die stark erweiterten und mit verdickten Wänden versehenen Capillaren hervortreten lässt. Die Nervenzellen sind trüb und verschmälert, was am besten in der Schicht der grossen Pyramidenzellen wahrzunehmen ist. Die markhaltigen Nervenfasern (Weigert-Pall'sche Färbung) sind gefärbt, aber nicht so gut, wie die eines gesunden Thieres. Den eben erwähnten Veränderungen begegnet man am Kleinhirn, wo nur die innerste Schicht sich scheinbar unverändert zeigt, an der mittleren dagegen erkennt man trübe Zellen, von denen einige atrophisch sind.

In der Medulla oblongata haben die Veränderungen ihren Sitz vorwiegend in der grauen Substanz. Die zelligen Bestandtheile nehmen die Farbe nicht mehr gut an, die Zahl der Ganglienzellen hat abgenommen, sie haben an Grösse eingebüsst, was deutlicher im Rückenmark zur Beobachtung kommt.

Das Rückenmark färbt sich im Allgemeinen schlecht. Die Pia mater (Halsgegend) ist verdickt, reich an Kemen und kleinen Rundzellen, die hie und da kleine Heerde bilden; man beobachtet einige kleine Hämorrhagien unter der Pia. Weiter erkennt man Vermehrung der Neuroglia- und Atrophie der Ganglionzellon, deren Zahl besonders in den vorderen Hörnern sehr vermindert ist. Manche Zellen haben ihre Ausläufer verloren und sich in unbestimmt begrenzte Massen umgewandelt, welche noch kernhaltig sein können; jedenfalls sind sie viel kleiner, als die ursprünglichen Ganglienzellen. Ein weiteres Stadium dieser Veränderung stellt der Zerfall der Zellen dar, von denen nur Spuren bleiben, indem an ihrer Stelle bald leere, bald mit Körnern gefüllte Vacuolen zur Beobachtung kommen. Die graue Substanz zeigt woisse, in's Gelbe fallende, körnige, aus Erweichung entstandene Heerde, welche ihren Sitz vorwiegend in der Nähe des Centralkanals haben. Im Centralkanal findet man eine körnige, stellenweise reticulär aussehende Substanz, welche einem eiweissartigen Exsudat ähnelt. Die cylindrischen Zellen, welche das Lumen des Contralkanals auskleiden, sind wie gequollen. Im Brust-, Lenden- und Sacraltheile wird die Hyperämie, sowohl in der weissen, als 
in der grauen Substanz, stärker. Im Lendentheile haben mehrere kleine Hämorrhagien stattgefunden, welche vorwiegend im vorderen rechten Horne ihren Sitz haben. Die Gefässe der grauen Substanz sind erweitert und der Art mit Blutkörperchen gefüllt, dass die kleinsten Gefässverästelungen besser zum Vorschein kommen. Die Capillaren zeigen in ihrem Innern die Blutkörperchen rosenkranzartig angeordnet, einige lassen in ihrer Umgebung zerstreute Blutkörperchen sehen.

Die Nervenzellen färben sich nicht mehr gut, sie sind verschmälert, atrophisch, einige haben ihre Fortsätze verloren und erscheinen fast rund, klein, körnig, was am deutlichsten in den vorderen Hörnern zu sehen ist. Das Aussehen der grauen Substanz weicht von dem normalen ab; an manchen Stellen, wo die Ganglienzellen zu Grunde gegangen sind, zeigt sie bei Anwendung schwacher Vergrösserung kleine Höhlen und Verschmälerung beider Vorderhörner; letzteres ist deutlicher im Lendentheile bei Vergleichung mit Querschnitten vom Rückenmarke eines gesunden Huhnes von gleichem Alter wahrzunehmen. Die Wucherung der Neuroglia, die Verdickung der Pia mater, die Hämorrhagien unter der Dura und der Arachnoides fehlen im Lendentheile nicht. Der Centralkanal bietet das nämliche, oben geschilderte histologische Bild. Im Lendentheile haben wir an vielen Schnitten eine gleichmässige Erweiterung des Kanals mit partiellem Verlust des Endothels und blutigem Inhalt beobachtet. In der weissen Substanz sieht man ausser der starken Congestion Wucherung der Neuroglia und Verschmälerung der Nervenfasern, welche sich nicht mehr gut nach der WeigertPall'schen Methode färben lassen (Taf. V. Fig. 1-4).

N. ischiadicus (Taf. V. Fig. 5-7). Da wir die peripherischen Nerven so frisch als möglich studiren wollten, so haben wir das Thier bald nach dem Tode obducirt und die Methode von Ranvier-M. Schultze angewandt. Im Querschnitt beobachteten wir eine Atrophie der markhaltigen Nervenfasern, eine Umgestaltung und zwar eine Fragmentation des Myelins, welches stellenweise körnig aussah. Die Zahl der Myelinkörner war mehr oder weniger gross, dieselben boten dem entsprechend eine verschiedene Grösse dar. An Zupfpräparaten, welche absichtlich hergestellt wurden, um die einzelnen Fasern in ihrer Länge zu 
durchmustern, kommt die verschieden schwarze Färbung der Nervenfasern im Vergleich mit der der normalen alsbald zum Vorschein. Die normalen Fasern tingiren sich in der That gleichmässig schwarz, diejenigen der verdursteten Thiere nehmen dagegen eine schwache, blasse, nicht homogene Färbung an, so dass die Markscheide an manchen Nervenfasern unregelmässige Anhäufungen und quer hindurchgehende, mehr oder weniger genäherte Brüche darbietet. Einzelne Körner sind an manchen Stellen nicht tiefschwarz (Osmiumsäure), sondern gräulich gefärbt. Diese sind bald vereinzelt, bald gruppenweise angeordnet; zwischen ihnen liegen helle Querzüge oder Räume. An einzelnen Nervenfasern beobachtet man die Bildung eines die oben geschilderten Körner enthaltenden weitmaschigen Netzes.

Der Axencylinder dieser Fasern ist verändert: er bietet nehmlich partielle Anschwellung und hie und da bedeutende Verunstaltung dar. Manche Nervenfasern sind ganz atrophisch; diese Atrophie zeigt sich besser an Schnitten, die aus Stücken stammen, welche in Müller'scher Flüssigkeit fixirt, dann in allmählich verstärktem Alkohol gehärtet und nach der Weigert-Pall'schen Methode gefärbt waren. An diesen Schnitten bemerkt man Hyperämie, Bindegewebsverdiokung und eine in verschiedenem Grade intensive Atrophie der Nervenfasern, deren Markscheide sich gar nicht mehr oder nur schwach und stellenweise färbt, so dass viele Fasern eine mehr oder weniger grosse Zahl von rosenkranzartig angeordneten dunklen Körnern in ihrem Innern zeigen (Ocul. 3, Obj. homog, Immers. $\frac{1}{18}$ Zeiss). Viele Fasern erscheinen weit von einander gelagert; der so entstandene $Z$ wischenraum wird von einer körnigen Substanz eingenommen, die, wie bei starker Vergrösserung ersichtlich, von entfärbten, sehr atrophischen Nervenfasern herrührt, welche stellenweise den gleichfalls veränderten Axencylinder darstellen.

Dieselben Resultate haben wir bei der Untersuchung des N. vagus und des N. glossopharyngeus erhalten, welche absichtlich in Bezug auf die locale Empfindung des Durstes untersucht wurden.

Im Herzen bemerkt man starke Congestion, welche die kleinsten Capillaren zierlich hervortreten lässt. An manchen Stellen 
haben kleine Hämorrhagien stattgefunden und die Muskelfasern, welche an diese hämorrhagischen Heerde grenzen, haben ihre normale Streifung theilweise eingebüsst. Zwischen den Fasern sieht man hie und da wenige Rundzellen, welche sich um die Venen zahlreicher finden. Die Arterien zeigen eine die ganze Gefässwand einnehmende Entzündung, die in der Intima am stärksten ausgeprägt ist.

An den Muskeln des Relationslebens haben wir dieselben Veränderungen wahrgenommen, aber hier sind die Hämorrhagien seltener und kleiner im Vergleich mit den im Herzen beobachteten. Das innere Perimysium ist mit Rundzellen infiltrirt, welche kleine Heerde bilden.

Aorta und rechte Carotis. Die Adventitia zeigt starke Hyperämie und kleine umschriebene Hämorrhagien; sie ist zugleich Sitz einer kleinrundzelligen Infiltration, welche, obgleich in geringerem Grade, über die Tunica media hinausgreift. Die Intima zeigt sich fast unverändert.

Kamm. Mikroskopisch erkennt man eine bedeutende Erweiterung der Gefässe, welche mit Blutkörperchen ganz gefüllt sind, so dass das Gewebe ein cavernöses Aussehen erbält. Das Bindegewebe ist reich an kleinen Rundzellen. Das Endothel zeigt sich trübe.

In den Lung en findet man allgemeine Congestion. Zwischen den Lungenläppchen, im interlobularen Bindegewebe und in den meisten Alveolen beobachtet man Blutkörperchen, so dass in den Schnitten der Inhalt der Alveolen aus einem, aus unmittelbar neben einander liegenden Blutkörperchen gebildeten Netze besteht; nur selten begegnet man einem leeren oder fast leeren Alveolus. Die Bronchien sind in Entzündung. In dem faserigen Bindegewebe, welches den ganzen Bronchus und die mit diesem verlaufenden Gefässe und Nerven umhüllt, befinden sich kleine zerstreute Rundzellen. Das interlobulare Bindegewebe und die Wände der Bronchien enthalten viel schwarzes Pigment.

Die Milz ist atrophisch. Die bindegewebige Hülle (die Kapsel) zeigt sich verdickt und an manchen Stellen ist sie Sitz einer kleinrundzelligen Infiltration. In der zunächst unter der Kapsel gelegenen Pulpa bemerkt man zahlreiche, meistens 
kleine, umschriebene Hämorrhagien, von denen einige grössere Heerde bilden. Dieselben Hämorrhagien, obgleich weniger zahlreich und kleine Heerde bildend, findet man in der Nähe der Balken um die Malpighi'schen Körperchen, vorwiegend zur Seite der Arterien, welche entzündliche Veränderungen ihrer Häute darbieten. Die Pulpa ist gering. Die Leukocyten bilden kleinere, nicht mehr dichte, kuglige Ballen, so dass die Malpighi'schen Körperchen verdünnt aussehen; es scheint sogar, dass sie an manchen Stellen verschwunden seien, da man eine grössere Zahl von kleinen Arterien im Vergleich mit Schnitten aus einer normalen Hühnermilz bemerkt. Die kleinen Arterien zeigen deutlich entzündliche Veränderungen, welche nicht nur die Intima, sondern auch, obgleich in geringem Grade, die übrigen Arterienhäute betreffen. In manchen Arterien ist das Lumen fast ganz durch ein Gerinnsel obturirt. Viele Pulpazellen befinden sich in indirecter Theilung.

Niere (Taf. VI. Fig. 4-6). Bei Untersuchung des frischen Organs bemerkten wir: fettig-körnige Metamorphose des Harnkanälchenepithels, welche in den gewundenen Kanälchen stärker ausgeprägt war; partielle Zerstörung des Epithels, die sich vorwiegend in den Sammelröhrchen zeigte; allgemeine starke Hyperämie und Blutungen in manchen Bowman'schen Kapselṇ und in den Röhrchen. Nachdem Nierenstücke in grossen Mengen von Müller'scher Flüssigkeit fixirt und nachfolgend in steigender Alkoholverstärkung gehärtet waren, machten wir aus denselben Schnitte. Wir haben ausser der oben erwähnten Hyperämie, welche die Capillaren am deutlichsten hervortreten lässt, eine kleinzellige Infiltration um die Gefässe, die Bowman'schen Kapseln und im lockeren interstitiellen. Bindegewebe beobachtet. Man findet weiter Blut im Kapselraum und in den Tubulis, von denen einige Blut-, andere Hyalin-, wieder andere, die grösseren, Epithelcylinder in ihrem Lumen enthalten. Manche Tubuli zeigen, indem ihr degenerirtes, von der Wand losgelöstes Epithel im Centrum gesammelt ist, Blutkörperchen zwischen dem so entstandenen Epitbelcylinder und der Wand. Im Allgemeinen ist das Epithel durch das Blut comprimirt, es erscheint deswegen blass, körniger, die Zellencontouren sind verschwommen, and die Zellenfärbung will nicht recht gelingen. Der Kapsel- 
raum enthält häufig abgestossenes Epithel, welches bald noch wohl erhalten, bald nekrotisch oder blasig degenerirt erscheint. Damit hängt die Erscheinung zusammen, dass durch Desquamation des degenerirten Epithels die Capillarschlingen zum Theil von ihrem Epithel entblösst sind; andere Glomeruli dagegen lassen eine in abnormer Mächtigkeit entwickelte Epithelbekleidung auf den Gefässschlingen sehen. Die Zellen der Tubuli zeigen indirecte Theilung (Taf. VI, Fig. 6).

Wichtiger ist die Staungshyperämie in der Leber, welche vermöge ihrer Circulationsverhältnisse dazu ganz besonders disponirt ist. Die Hyperämie betrifft hauptsächlich die Centralvene und die in sie mündenden Capillaren. Die Leberzellenbalken zeigen eine Verschmälerung, welche dem ausgeäbten Blatdrucke zuzuschreiben ist. Die Leberzellen sind im Allgemeinen kleiner, weit von einander gelegen, ihr noch vorhandener Nucleus nimmt aber nicht mehr gut die Carminfarbe an. Um die grossen Venen bemerkt man eine in's Gelbe fallende Schicht, die aus den anliegenden, yon der Vene selbst ausgedrückten Leberzellen gebildet wird. Die Glisson'sche Kapsel ist mit kleinen Rundzellen hie und da infiltrirt. Diese kleinzelligen Elemente befinden sich zahlreicher in der Nähe derjenigen Stellen, wo kleine Hämorrhagien unter der bindegewebigen Kapsel stattgefunden haben. Das interlobulare Gewebe scheint hie und da vermehrt. Die Gallencapillaren bieten keine wahrnehmbare Veränderung. Spärliche Leberzellen zeigen Mitosen (Taf. VI, Fig. 7). In der Zunge bemerkt man eine starke Stauungshyperämie. Die oberflächlichsten Muskelbündel erscheinen blass. Die diese Bündel in verschiedener Anzahl bildenden Fasern haben ihre dunklen und hellen Querbänder theilweise eingebüsst, sie erscheinen getrübt und wie getrocknet. In dem knorpeligen Theile der Zunge sind die Zellen stark verändert, sie füllen die Höhlen der Grundsubstanz unvollkommen aus. Diese atrophischen Knorpelzellen lassen zackige Contouren erkennen und gleichen den stechapfelförmigen Blutkörperchen nach Einwirkung einer intensiven Leydener Flasche. Diese zackige Veränderung ist aber nicht in der ganzen Grenzlinie der Zellen wahrzunehmen, sondern die Zacken sind bald nur auf eine Seite beschränkt, bald nehmen sie die ganze Peripherie ein; in beiden Fällen Arehiv f. pathol. Anat. Bd. 139. Hft. 1. 
zeigen sie sich ungleichmässig vertheilt und verschieden hervorragend. Der Zellkern ist kleiner, nicht gut gefärbt (Saffranin, Gentianaviolett u. s. w.). Manche kleine Knorpelzellen sind sehr blass, ihre Kerne nehmen nicht mehr gut die Farben an. In den Zellen, wo die Atrophie stärker ausgeprägt ist, sieht man an einer Stelle der Knorpelkapsel Reste des manchmal mit Kernen versehenen Zellprotoplasmas haften bleiben. Viele Kapseln zeigen sich verkleinert und leer, andere dagegen enthaiten Zellen mit durchsichtigem Protoplasma und kaum wahrnehmbarem Kerne, der sich ans einer leichten Schattirung erkennen lässt; wieder andere bieten an der Peripherie einen aus stark gefärbten Körnern gebildeten Ring (Methylviolett B) und sind auch mit den oben geschilderten Zacken versehen. Manche Knorpelkapseln, welche ein leeres oder fast ganz leeres lumen zeigen, enthalten in ihrem Innern eine Anzahl von verschieden grossen Körnern, welche der Peripherie entlang aufgereiht erscheinen (Taf. VI, Fig. 3).

Kropf. Die Stauungshyperämie tritt hier charakteristisch hervor. Die Röthung ist fleckig, mit kleinen oberflächlichen Blutungen in der Schleimhaut verbunden, deren zottenartige Vorsprünge zwischen den Magengrübchen durch diese Blatanhäufung vergrössert erscheinen und mehr über die Oberfläche hervorragen. Die Schleimhautoberfläche, wo dieselbe noch mit Epithel versehen ist, zeigt eine glasige dünne Schicht von Exsudat, welche durch Blutbeimischung etwas röthlich aussieht; sie enthält Kerne und Rundzellen. Einige Drüsen haben einen blasenförmig erweiterten Grund, sie enthalten eine feinkörnige Substanz, welche hie und da abgestossene veränderte Drüsenzellen einschliesst. Andere Drüsenzellen, welche sich noch an ihrem rechten Platze befinden, zeigen sich abgeplattet und atrophisch. Man bemerkt weiter eine geringe kleinrundzellige Infiltration, welche in der Mucosa und $z$ wischen den Fasern der Muskelhaut, vorwiegend um die Venen herum, ihren Sitz hat. Die Muskelhaut ist blass, trübe, die Faserkerne färben sich schlecht. Das blasenförmige Aussehen der Kropfdrüsen erinnert an das Anfangsstadium der cystischen Erweiterung.

Darm. Was über die Circulationsstörungen am Magen gesagt wurde, gilt in gleicher Weise auch für den Darm. Hier 
ist aber noch zu bemerken, dass die Stauungshyperämie geringer ist und dass Blutungen in den Solitärknötchen stattgehabt haben. Das Drüsenepithel ist kleiner, als normal; das zwischen den Drüsen, unter denselben und zwischen den Fasern der Muskelhaut vorhandene Gewebe ist Sitz einer kleinrundzelligen Infiltration. Die Zellen, sowohl des Kropfes, wie des Darmes zeigen Mitosen.

Die Hoden sind hyperämisch. Die Tubuluszellen sind verkleinert, atrophisch, und färben sich mit Carmin schlecht; die Tubuli selbst sind im Ganzen enger. Die Samenfäden fehlen vollständig, auch fehlt jede Spur von indirecter Theilung der Spermatogonien (Stammzellen). Die verdickte bindegewebige Hülle (die Tunica albuginea s. fibrosa) ist Sitz einer kleinzelligen Infiltration, welche weiter längs der Septula testis hinein vordringt und auf diese Weise die pyramidalen Läppchen umhülltt.

Die Lymphdrüsen zeigen Atrophie der Marksubstanz und Verdickung der Trabekel. Die Blutgefässe sind erweitert und mit Blut gefüllt. Die faserige Kapsel erscheint verdickt.

Knochen. Keine Veränderung bietet die Haut und die eigentliche Knochensubstanz, nur das Knochenmark zeigt bei makroskopischer Betrachtung eine dunkelrothe Farbe.

Schlüsse.

Die Ergebnisse unserer Versuche beweisen den grossen Werth des Wassers für das Leben. Die Thiere, welche des Wassers beraubt wurden, gingen nach wenigen Tagen zu Grunde. Bei unserem Hunde trat der Tod ungefähr am 11. Tage nach der Wasserentziehung ein, nachdem er 24,25 pCt. seines Körpergewichts verloren hatte. In den letzten Lebenstagen hatte er die Hungerempfindung vollständig verloren, indem er keine dargebotene feste Nahrung anrührte; die Hündin dagegen (Luciani und Bufalini), welche nur der festen Nahrung beraubt wurde und eine tägliche Wasserdosis von $150 \mathrm{~cm}$ bekam, lebte 43 Tage.

Die Resultate stimmen mit den im December 1886 von Lebard veröffentlichten äberein. Allgemeine Kraftlosigkeit, höchste Mattigkeit, Kreislaufs- und Athmungsstörungen, qualitative und quantitative Veränderungen des Harns, die numerische 
Veränderung des Blutes u. s. w. folgen schnell auf einander. Unsere Hühner starben zwischen dem 8. und 10. Tage nach der Wasserentziehung, sie frassen aber beständig, wie es der Inhalt des Kropfes bewies, bis zum letzten Lebenstage; sie verloren in so kurzer Zeit 33,83-41,23 pCt. ihres Anfangsgewichtes. Dieser Gewichtverlust erscheint noch bedeutender, wenn man in Betracht zieht, dass unsere Thiere täglich Maiskörner gefressen haben, wie man aus der Tab. II ersieht. Dies beweist noch mehr den höchsten Einfluss, welchen das Wasser auf die allgemeine Ernährung ausübt, und wie die Abzehrung der Gewebe den täglichen Mangel an Wasser bis zu einem mit dem Thierleben zulässigen Grade ersetzt.

Bei den Hühnern, welche bis zum Tode frassen und nicht, wie der Hund, in vollständiger Inanition zu Grunde gingen, epgab die histologische Untersuchung wichtige Resultate. Die in verschiedener Intensität, je nach dem Organe ausgeprägten Veränderungen könven nicht leicht und genau kurz zusammengefasst werden, deshalb verweisen wir auf das oben Gesagte.

An den Leichen von Hühnern, an welchen mit blossem Auge Abmagerung, Austrocknung der verschiedenen Organe, Stauungshyperämie deutlich war, bemerkten wir bei der histologischen Untersuchung kleinzellige Infiltration des Bindegewebes, Hämorrhagien in der Zunge, in den Muskeln des Relationslebens, im Herzen, in den Lungen, der Milz, Leber, im Kropfe, in den Nieren, im Nervensystem u. s. w.

Die Zellen waren atrophisch und theilweise verschwunden: dies trat besonders deutlich in der Milz, im Zungenknorpel und im Nervensystem hervor. In diesem letzten fanden wir Atrophie der anatomischen Elemente, besonders der Ganglienzellen der Vorderhörner des Rückenmarkes, eine Andeutung degenerativer Reaction der Markfasern, Proliferation in der Neuroglia, Hyperplasie der Pia mater, Hämorrhagien unter der Arachnoides und Dura, Ependymitis und Hämorrhagie im Centralkanale; in den peripherischen Nerven fanden sich deutliche Veränderungen mit Atrophie und Neuritis parenchymatosa. Im Nervensystem war die Abnahme und das vollständige Verschwinden einzelner Markfasern auffällig. Diese letztere Veränderung muss gewiss einen schlechten Einfluss auf den Zustand der anderen Organe 
ausüben und schnell den Tod der Versuchsthiere herbeiführen. Solche Erscheinungen zeigen sich neben Bindegewebshyperplasie und Zellenneubildung durch Karyokinese.

Diese allgemeine Atrophie und das Verschwinden der Zellen erklärt den bedeutenden und schnellen Verlust des Anfangsgewichtes des Thierkörpers und bestätigt unserer Meinung nach den Schiff'schen Schluss, der die Durstempfindang zu den Gemeingefühlen rechnet, indem er jede Beziehung zu besonderen Nerven leugnet.

Man empfindet den Durst im Pharynx, im Munde, besonders im hinteren Theile der Zunge, weil diese_ Regionen einer stärkeren Luftwirkung ausgesetzt sind und so schneller Trockenheit in denselben sich einstellt; deshalb brauchen sie mehr Wasser, als andere Theile des Körpers. Diese Meinung wird durch den beträchtlicheren Grad der Zungenveränderungen bekräftigt, welche ihre Höhe in dem knorpeligen Theile erreichen. Ausserdem bietet die Nervenuntersuchung dieser Region die nämlichen Veränderungen, und zwar in derselben Intensität, dar, welche in anderen Nerven beobachtet worden sind. Uebrigens ist es bekannt, dass die Durchschneidung des N. glosso-pharyngeus, des $N$. vagus und des $N$. lingualis die Durstempfindung nicht aufhebt.

Diese Versuche beweisen, dass der Durst nicht eine auf das eine oder andere Organ beschränkte, sondern eive allgemeine Empfindung darstellt, welche dann auftritt, wenn das Wasser der anatomischen Elemente bis auf einen Minimalgehalt, der aber das Leben erlaubt, reducirt wird. Die gestörte Thätigkeit der einzelnen Zellen veranlasst die Störung der Function der verschiedenen Organe, des ganzen Organismus und endlich den Tod.

Als eine weitere Ursache des Todes muss die Zurückhaltung der Zersetzungsstoffe, wovon der Organismus durch verschiedene Se- und Excrete sich befreit, in Betracht gezogen werden. In der That wird dies durch die Versuche Nothwang's bestätigt, der bewies; dass die verdursteten Thiere mehr Auswurfsstoffe, als die gesunden, enthalten, was von Pernice und Alessi schon früher gefunden worden war.

Es versteht sich, dass die Eindickung des Blutes, die Austrocknung der Haut, der Schleimhäute u. s. w. und die geringe Harnausscheidung eine Zurückhaltung und Anhäufung von Zersetzungs- 
produkten herbeiführen müssen, weil diese nicht mehr genügend abgeführt werden können; somit wirken sie schädigend auf die Ernährung und die Leistung der zelligen Elemente. Die Organveränderungen können hierbei zum Theil mit Recht als Folge dieser Anhäufung von Zersetzungsprodukten aufgefasst werden.

Die oben geschilderte Glomerulonephritis haemorrhagica ist toxischer Natur, die Veränderungen des Kropfes dagegen glauben wir theilweise der mechanischen Wirkung der Maiskörner zuschreiben zu müssen. In der That werden die Maiskörner nicht genügend durch den Kropfsaft verändert, weil vielleicht den Zellen die Fähigkeit abgeht, einen normalen Saft zu bereiten, oder weil der auf der Schleimhautoberfläche sitzende Schleim den Uebertritt des Saftes von den Drüsen in den Magen hindert, oder auch weil geringe oder gar keine Absonderung desselben statthat. Die Erweiterung und Verlängerung der Drüsen möchten wir als Folge einer leichten Secretstauung in Folge der Vorengerung der Drüsenausführungsgänge ansehen.

Die Folgen der Wasserentziehung bei den Thieren sind also Kreislaufsstörungen, Gewebsatrophie, mehr oder weniger ausgeprägte entzündliche Organveränderungen, und dies Alles ist die Ursache des Todes.

$$
\text { L i t e r a t u r. }
$$

F. Tiedemann, Physiologie des Menschen. Bd. IIr. 1836.

Th. Scheffer, De animalium, aqua iis adempta, nutritione. Dissert. inaug Marburg 1852.

M. Schiff, Leçons sur la Pbysiologie de la digestion. 1867.

Lussana, Manuale di Fisiologia. 1868.

Meyer Jacques, Ueber den Einfluss vermehrter Wasserzufuhr anf del Stoffumsatz im Thierkörper. Centralbl. f. die med. Wissensch. $188 \mathrm{C}$ W und $\mathrm{t}$, Fisiologia umana. 1881.

Maass, Deber den Einfluss rapider Wasserentziebung auf den Organism u. s. w. Chirurgen-Congress, I. Jahresbericht für 1881.

L. Luciani e G. Bufalini, Sul decorso dell' inanizione. Archivio ,

Bizzozero. 1882.

G. Gaglio, Influenza dell' inanizione sulla struttura del fegato e del stomaco. Arehivio di Bizzozero. 1884.

v. Voit, Nittheilungen aus der Pfeuffer'schen Klinik. Epidemische Chole von Prof. Buhl. Zeitsehr, f. rationelle Med. N. F. Bd. VI. $18 \varepsilon$

P. J.Kappes, Ueber Wasserentziehung. Inaug.-Diss. Jahresbericht 18\{ 
Groll, Untersuchung über den Hämoglobingebalt des Blutes bei vollständiger Inanition. Diss. Königsberg. Jahresbericht 1888.

A. Gürber, Dje Gesammtzahl der Blutkörperchen und ibre Variation, Zahlenwerthe zum Oecus des Frosches. Arch. f. Anat. u. Physiol. Leipzig 1889.

Longet, Physiologie.

Ziegler, Lehrbuch der allg. pathol. Anat. 1890.

B. Morpurgo, Della neoproduzione di elementi cellulari nei tessuti di animali nutriti dopo un lungo digiuno. Archivio di Bizzozero. 1890.

Landois, Lehrbuch der Physiologie des Menschen. 1891.

Birch-Hirschfeld, Allg. Pathol. 1892.

Luciani, Fisiologia del digiuno. Arch. ital. di Biologia.

Pfeffers Pflanzenphysiologie. Bd. II. S. 450.

Falk und Scheffer, Der Stoffwechsel im Körper durstender, durststillender und verdunstender Vögel. Arch. f. physiol. Heilkunde. 13. Jahrgang.

C. Lehmann, F. Müller, I. Munk, H. Senator, N. Zuntz, Untersuchungen an zwei hungernden Menschen. Dieses Archiv, Supplementheft zum 131. Bd.

\section{Erklärung der Abbildungen.}

Tafel V.

Fig. 1. Quersehnitt durch den Brusttheil des Rückenmarkes. Graue Substanz. Färbung mit Hämatoxylin-Eosin. Man sieht in den vorderen Hörnern Vacuolen a in Folge ron Verschwinden der Ganglienzellen. Im Centralkanal findet man eine feinkörnige Substanz b. c stark gefüllte Blutgefässe. d Erweichungsheerde. Vergrösserung Obj. aa, Oc. 2 Zeiss.

Fig. 2. Querschnitt aus dem Lendentheil des Rückenmarkes. Graue Substanz. Das vordere Horn a ist mebr atrophisch, als das hintere. Vergrösserung Obj. a a, Oc. 2 Zeiss.

Fig. 3. Querschnitt aus dem vorderen Horne des Rückenmarkes (Brusttheil). a, a Vacuolen in Folge des Verschwindens der Ganglienzellen. b grosse Ganglienzelle in beginnender Atrophie und von einem ungefärbten Hofe umgeben. $b^{\prime}$ atrophische Ganglienzellen. c thrombosirtes Gefäss. d gewucherte Neuroglia. e, e degenerirte Nervenfasern. Färbung nach der Methode von Pall. 3ōomal vergrössert.

Fig. 4. Ein Theil des vorderen Hornes des Lendenrückenmarkes. a, a Vacuolen. b atrophische Ganglienzellen, c gewucherte Neuroglia. Färbung nach der Methode von Pall. 355mal vergrössert.

Fig. 5. Querschnitt des N. ischiadicus. Ausser Hyperämie bemerkt man eine Verdickung des interstitiellen Bindegewebes und Atrophie der Nervenfasern. Die Markscheide färbt sich șchwach oder gar nicht. Färbung nach der Hethode von Pall. $810 \mathrm{mal}$ vergrössert. 
Fig. 6. Nervenfasern aus einem Zupfpräparate des N. ischiadicus. Das Myelin ist fast verschwunden und die Nervenfasern sind kleiner. Färbung nach der Methode von Pall. 355mal vergrössert.

Fig. 7. Die Figur entstamıt dem in Fig. 6 in schwacher Vergrösserung gezeichneten Präparat. In A tritt die graue Farbe des Myelins stellenweise hervor, in B ist das Myelin bedeutend geschwunden und der Axencylinder zeigt ein kolhig angeschwollenes Knde. 810mal vergrössert.

\section{Tafel VI.}

Fig. 1. Grund cystisch erweiterter Pepsindrüsen. a, a körniger Inbalt. a' körniger Inhalt, der desquamirte Zellen einschliesst. b kleinrundzellige Infiltration. $355 \mathrm{mal}$ vergrössert.

Fig. 2. Schleimdrüsen der Zunge. a blasenartige Epithelien. b reichlieher Inhalt. c kleinzellige Infiltration in interstitiellen Bindegewebe. 355 mal vergrössert.

Fig. 3. Atrophisehe Zellen des knorpligen Theils der Zunge. 810mal vergrössert. Erklärung im Text.

Fig. 4. Niere. a Glomerulitis. a' Capsulitis. b, b, b, b Harnkanälehen mit verfettetem Epithel. $b^{\prime}$ abgestossenes Epithel. b' körnige Cylinder. c schwache kleinrundzellige Infiltration im intertubularen Bindegewebe. Vergrösserung 540.

Fig. 5. Ein Theil eines geraden Harnkanälchens. Die Epithelzellen zeigen sich fettig degenerirt. Fixation nach Flemming. Färbung mit Saffranin. 540mal vergrössert.

Fig. 6. Ein Theil eines Harnkanälehens. Zellen in indirecter Theilung. Fixation nach Flemming. Färbung mit Saffranin. Zeiss, bomog. Immersion $\frac{1}{18}$, Oc. 2.

Fig. 7. Portales Bindegewebe. a prall gefüllte Vena interlobularis. b schwache kleinrundzellige Infiltration um die Gallengänge. 